\title{
gु \\ Dynamics of dark-soliton formation in a polariton quantum fluid
}

\author{
G. Grosso, G. Nardin, ${ }^{*}$ F. Morier-Genoud, Y. Léger, ${ }^{\dagger}$ and B. Deveaud-Plédran \\ ICMP, Ecole Polytechnique Fédérale de Lausanne (EPFL), 1015 Lausanne, Switzerland \\ (Received 29 March 2012; revised manuscript received 19 June 2012; published 30 July 2012)
}

\begin{abstract}
Polariton fluids have revealed huge potentialities in order to investigate the properties of bosonic fluids at the quantum scale. Among those properties, the opportunity to create dark as well as bright solitons has been demonstrated recently. In the present experiments, we image the formation dynamics of oblique dark solitons. They nucleate in the wake of an engineered attractive potential that perturbs the polariton quantum fluid. Thanks to time and phase measurements, we assess quantitatively the formation process. The formation velocity is observed to increase with increasing distance between the flow injection point and the obstacle which modulates the density distribution of the polariton fluid. We propose an explanation in terms of the increased resistance to the flow and of the conditions for the convective instability of dark solitons. By using an iterative solution of the generalized Gross-Pitaevskii equation, we are able to reproduce qualitatively our experimental results.
\end{abstract}

DOI: 10.1103/PhysRevB.86.020509

PACS number(s): 67.10.Jn, 71.36.+c, 03.75.Lm, 78.67.-n

In the recent past much effort has been devoted to characterizing the properties of quantum fluids, with particular attention given to phase transitions such as the Bose-Einstein condensate (BEC) and superfluidity. As in everyday fluids, waves and turbulence are also expected at the quantum scale. Quantized vortices ${ }^{1,2}$ and spin texture, ${ }^{3}$ for example, have been topics of major discussion. Presently, there is growing interest around solitons, especially in condensed matter systems. ${ }^{4,5}$ Solitons are solitary waves which propagate in the medium while maintaining their shape. The stability of their shape is the result of the exact compensation of the dispersion by the interparticle interactions. For attractive interactions, bright solitons (BSs) are formed. ${ }^{5}$ For the opposite case of repulsion, dark solitons (DSs) may appear, having the shape of density depressions in the fluid.

Originally predicted in the $1970 \mathrm{~s},{ }^{6}$ DSs have been experimentally observed only 20 years later in the field of nonlinear optics ${ }^{7}$ and then in cold atom BECs by phase and density imprinting. ${ }^{8,9}$ DSs are indeed characterized by both a density minimum and an associated phase shift. ${ }^{10}$ However, imprinting is not the only possible way to create a DS. More recently, the growing attention to quantum hydrodynamics has triggered a clear interest towards the nucleation of DSs in the wake of an obstacle. Similar to a boat sailing across calm waters, an obstacle flowing in a quantum fluid can leave turbulence in its wake ${ }^{1}$ and generate waves. Under particular conditions, solitons can form in the condensate. ${ }^{2}$ Such conditions are basically set out by the density and the velocity of the fluid, together with the nature of the obstacle. Recently, condensed matter systems, and in particular exciton-polaritons, have turned out to be a very accessible means to study quantum hydrodynamics. Polaritons are half-matter half-light particles arising from the strong coupling between excitons and cavity photons in a semiconductor microcavity. They have evidenced $\mathrm{BEC}^{11}$ as well as superfluidity. ${ }^{12}$ Additionally, polaritons can be easily optically manipulated and controlled, and this promotes them to the frontline in order to study quantum hydrodynamics. Recently it has been proven that under certain conditions quantized vortex pairs ${ }^{13,14}$ and dark solitons ${ }^{4}$ can be nucleated when a polariton quantum fluid scatters against a defect.
Although quite exhaustive information is available regarding vortices, only little information is available on the dynamics and the formation of DSs. The cause of this absence may be found in the difficulty of finding a proper theoretical approach to the transient between the time at which the flow is switched on and the time needed to reach a stationary state. Experimentally also the study of the transient is arduous because phase and time resolved imaging of the fluid is required, which is not so immediate. The formation process of DSs is of crucial importance for all those fields in which stable hydrodynamic solitons and strict control of their propagation are required.

In our experiments we create dark solitons by perturbing the polariton fluid with an obstacle, and we aim at investigating the formation dynamics of hydrodynamically nucleated dark solitons. The phase and amplitude of polaritons are time resolved through a homodyne detection system. This is achieved by recording the spatial interference between the polariton emission and the local oscillator, at the output of a Mach-Zehnder interferometer. ${ }^{13,15,16}$ Polaritons are resonantly injected in a GaAs microcavity by means of a 2 ps long pulse with a given in-plane wave vector. The resulting polariton wave packet is then free to evolve in time. The number of particles composing the fluid is simply controlled by the pump power. In-plane momentum and fluid density are the most relevant parameters for hydrodynamic behavior since they directly set the Mach number $(M)$ of the fluid. $M$ is the ratio between the flow velocity $v_{\text {flow }}$ and the speed of sound $c_{s}$ in the fluid, the latter being directly related to the fluid density. For $M$ around 1 , vortex-antivortex pairs are nucleated in the perimeter of the obstacle, ${ }^{13}$ whereas for larger values of $M$, dark solitons have been observed to decay into vortex streets. ${ }^{15}$ Moreover, DSs are found to be more stable when decreasing the pump power. Therefore, control of the polariton density distribution is necessary in order to create and study DSs. Such fine tuning within the window in which DSs are stable is challenging and is achieved experimentally by moving the laser spot with respect to the obstacle position. In the experiments the laser spot has a Gaussian profile with a FWHM of $15 \mu \mathrm{m}$, and the power is kept to $5 \mathrm{~mW}$ during the whole set of experiments. These parameters give an intensity change of a few percents for a 


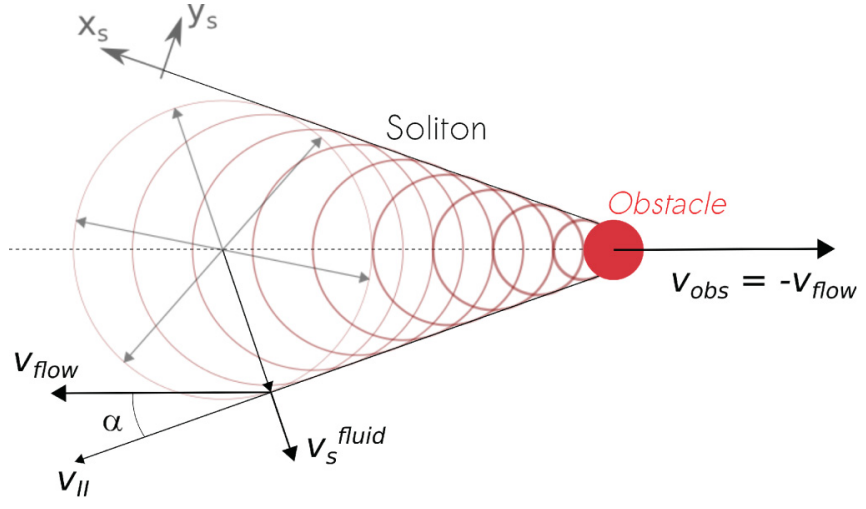

FIG. 1. (Color online) Dynamics of a 2D fluid passing an obstacle in the fluid frame, showing the formation of dark solitons. The obstacle moves with $\vec{v}_{\text {flow }}$, leaving behind perturbations which expand as circular waves and form dark solitons by tangential interference. The latter move in the $y_{s}$ direction with respect to the fluid frame, whereas they grow along the $x_{s}$ direction in the obstacle frame.

distance of $5 \mu \mathrm{m}$ from the laser spot center. Intensity tuning of the order of tenths of $\mu \mathrm{W} / \mu \mathrm{m}^{2}$ is achieved by moving the laser spot by half a micron. This is an extremely fine tuning which is hardly achievable by simply changing the pump power of the laser by means of a neutral density filter. The dependence of the different hydrodynamic regimes induced by specific excitation profiles with respect to the obstacle has already been highlighted in Ref. 17. We will show further that the density distribution strongly impacts also the DS formation velocity. The polariton fluid is perturbed by using an engineered negative potential provided by a mesa in the microcavity plane. Mesas are realized by etching the spacer before growing the top distributed Bragg reflection (DBR). ${ }^{18}$ This results in attractive potentials for polaritons, which are able to confine polaritons, as well as to perturb the flow of the two-dimensional (2D) polariton fluid in the cavity.

The dynamics of a 2D fluid passing an obstacle and the soliton formation can be analyzed both in the laboratory and in the fluid reference frame. In the latter, the obstacle moves with a velocity $\vec{v}_{\text {obs }}=-\vec{v}_{\text {flow }}$, perturbing the flow, and leaving behind circular waves which expand radially, in analogy to one-dimensional (1D) solitons (red circles in Fig. 1). Positive interference between such waves occurs tangentially with respect to the circular wave fronts. The resulting oblique soliton velocity in the fluid frame, $v_{s}$, is the only surviving component of the expansion of perturbation waves (gray arrows in Fig. 1). The presence of circular waves at the end of the soliton pattern has been revealed in a number of numerical simulations for different systems. ${ }^{2,19}$ In the fluid reference frame, 2D dark solitons move in the $y_{s}$ direction with a constant velocity given by $v_{s}=v_{\text {flow }} \sin (\alpha) .{ }^{4}$ Here, $\alpha$ is the aperture angle between a soliton and the motion of the mesa in the flow. In the laboratory frame (in which the obstacle is at rest) the component for the flow velocity must be added to the soliton velocity, annihilating the soliton orthogonal velocity.

Figure 2(a) shows the dynamics of dark-soliton formation. A polariton wave packet is injected at $t=0$ with an initial momentum of $1.5 \mu \mathrm{m}^{-1}$ in the vicinity of a $3 \mu \mathrm{m}$ wide mesa (circles). A few picoseconds after the injection, DSs begin to nucleate and their length grows. This is notable from the snapshots at different times in which the soliton length (dashed arrows) is clearly increasing within the 6 ps soliton time window. DSs are characterized by a density minimum $n_{\min }$ at the core within a surrounding fluid density $n_{0}$. The depth of DSs is then given by $n_{s}=n_{0}-n_{\min }$ and is related to the soliton velocity in the fluid frame through the formula for one-dimensional solitons, $v_{s} / c_{s}=\sqrt{1-n_{s} / n_{0}}$. Figure 2(b) shows an example of the polariton phase at $7.5 \mathrm{ps}$ after the injection: The phase jump due to the presence of the DS is clearly visible. The two dark solitons of Fig. 2(a) form with a different velocity. This asymmetry is explained by the different local conditions due to the position of the mesa with respect to the excitation spot and the different landscape of the photonic disorder. This aspect will be discuss later on. Confined states close to the continuum are also excited inside the mesa due to the broad energy spectrum of the pump. ${ }^{20}$ They appear as bright lobes in Fig. 2(a). In Fig. 2(c) we analyze the time dependent polariton density along $x_{s}$. Two wave fronts are visible. The first one, further from the mesa, is the front of the polariton wave packet expanding after the injection. The slope of this wave front in the density map represents $v_{\|}$, the velocity of the flow projected on $x_{s}$. This must be compared with the velocity extracted from the injecting momentum whose projection corresponds to a $v_{\|}=0.95 \mu \mathrm{m} / \mathrm{ps}^{15}$ In good agreement, the velocity measured in this case is $\sim 1.05 \mu \mathrm{m} / \mathrm{ps}$. The other wave front, the one closest to the mesa, corresponds to the expansion of the dark soliton and therefore its slope provides the formation velocity $v_{\text {form }}$ of DSs. Differently from our previous works, ${ }^{15}$ DSs are nucleated with a velocity appreciably smaller than the flow. Density profiles along $x_{s}$ are plotted for different times in Fig. 2(d).

A possible scenario allowing us to understand the origin of the formation velocity can be depicted assuming the formation velocity to be dependent on the particle density distribution during the soliton expansion. Intuitively, the more particles there are in the path of the DS, the harder it will be for the soliton dip to expand by pushing through the fluid. To check this hypothesis, we performed the same experiment for different positions of the laser spot with respect to the mesa and we measured the velocity of formation whenever possible. Experimentally, DSs are observed to form almost as fast as the flow velocity when the distance $d$ between the center of the mesa and the center of the excitation spot is larger than $7 \mu \mathrm{m}$. In all other cases, $v_{\text {form }}$ and $v_{\text {flow }}$ can be measured. The latter has an almost constant value ranging from 1.05 to $1.10 \mu \mathrm{m} / \mathrm{ps}$ for the experimental conditions studied. Experiments show that the formation velocity increases by increasing $d$. This observation also suggests a relation between the soliton formation dynamics and the local density distribution. In order to assess these local conditions, let us introduce another important parameter, the DS relative depth $\frac{n_{s}}{n_{0}}$. Figure 3 shows the changes of the ratio $\frac{n_{s}}{n_{0}}$ in time for different excitation conditions. The soliton amplitude $n_{s}$ has been calculated by averaging the density along the whole soliton length, whereas the density of the fluid $n_{0}$ is estimated from the maximum intensity, as shown in Fig. 2(d). Error bars indicate the standard deviation on the average. The solid lines of Fig. 3 correspond to distinct distances $d$ while the dashed line corresponds to the short soliton for the case $d=4.5 \mu \mathrm{m}$. The inset of Fig. 3 shows 
(a)
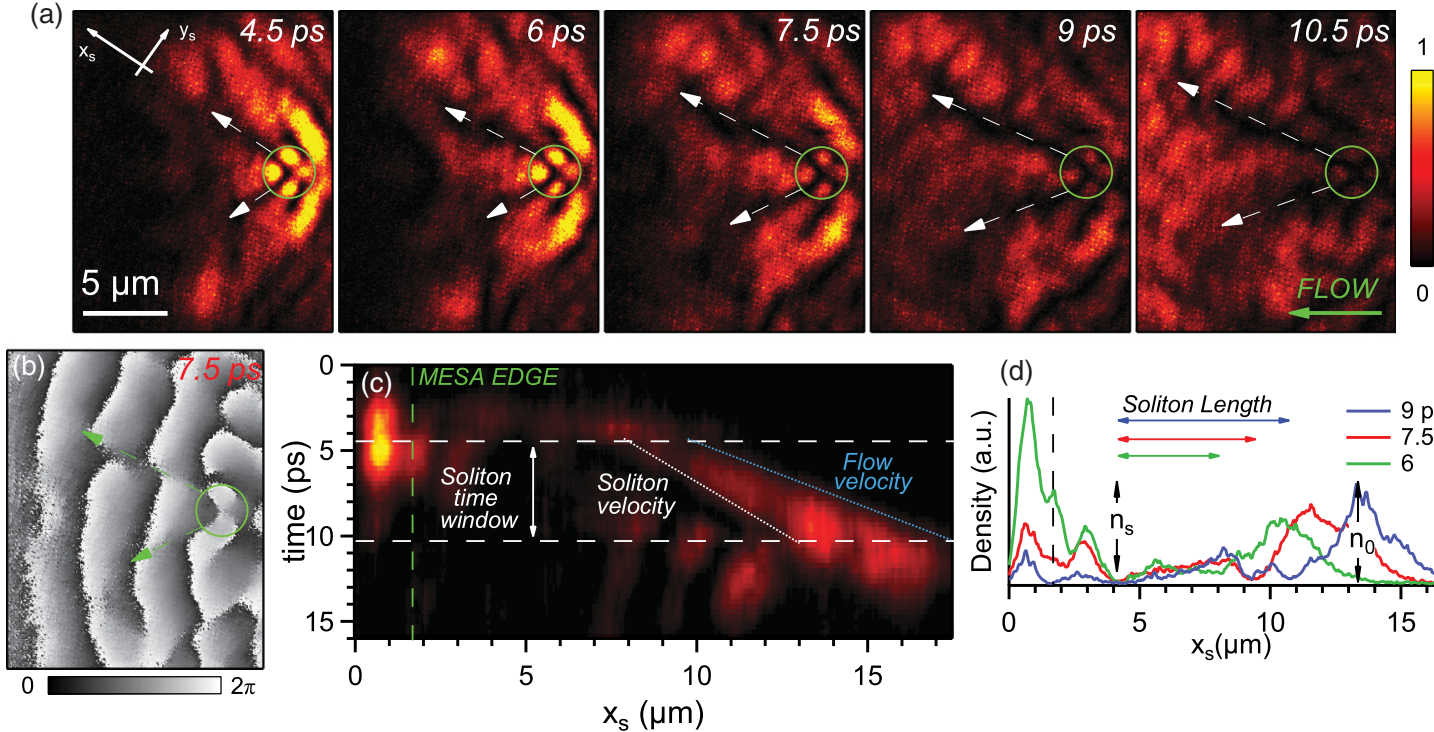

(d)

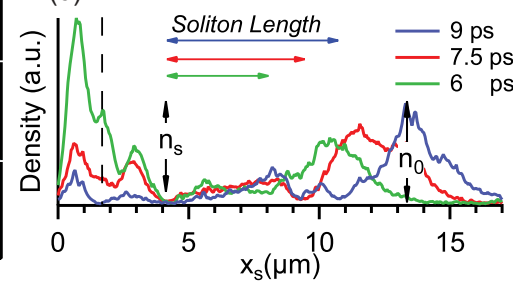

$\mathrm{x}_{\mathrm{s}}(\mu \mathrm{m})$

FIG. 2. (Color online) (a) Time evolution of the density of a polariton wave packet scattering on an engineered circular obstacle (green circle) of $3 \mu \mathrm{m}$ diameter. Polaritons are injected at $t=0 \mathrm{ps}$ with a $1.5 \mu \mathrm{m}^{-1}$ initial momentum in the vicinity of the mesa. The soliton dynamics is highlighted by white arrows. (b) Phase map of the polariton fluid 7.5 ps after the polariton injection, showing phase jump typical of DSs. (c) Density profile map along the direction of soliton growth $x_{s}$ vs time. (d) Density profiles at different times showing the growth of the DS along $x_{s}$.

the variation of the relative formation velocity with respect to the mesa-laser distance. Relating the results of Fig. 3 with the one of the inset, we can conclude that larger values of $\frac{n_{s}}{n_{0}}$ result in smaller formation velocities. A simple consideration can be used to understand this relation. In fact, large values of the ratio correspond to a strong polariton density gradient at the end of the soliton. This gradient tends to fill the low density region. At the same time, the DS tends to expand in the opposite direction due to the kinetic term imprinted by the flow. The growth rate of DS is the result of the interplay between these two terms. Figure 3 also shows that $\frac{n_{s}}{n_{0}}$ is overall larger when the laser spot is closer to the obstacle. The different spot positions give different density distributions and lead to different formation velocities, due to different local conditions during the formation dynamics. This is confirmed in the case of the short DS observed for $d=4.5 \mu \mathrm{m}$. As discussed previously, two solitons are generated for each

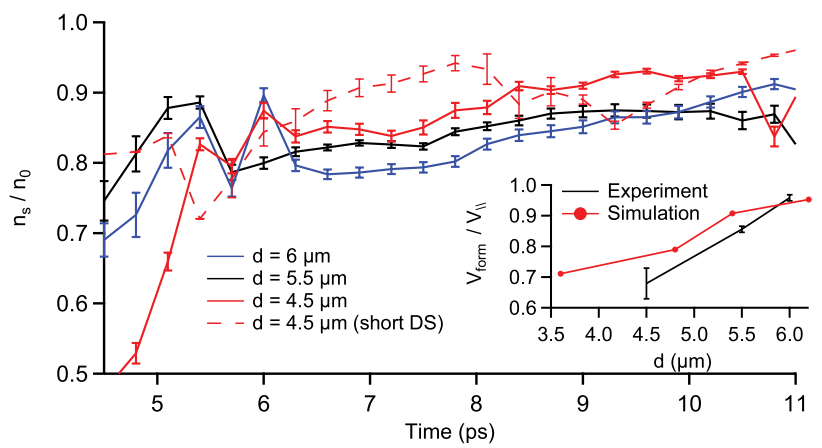

FIG. 3. (Color online) Time evolution of the ratio $\frac{n_{s}}{n_{0}}$ for different experimental conditions. The inset is the variation of the relative soliton formation velocity $\frac{v_{\text {form }}}{v_{\|}}$with the distance between the laser spot and the mesa. laser-mesa distance. For this specific case, the $v_{\text {form }}$ has been measured to be smaller than for the long soliton $(0.63 \mu \mathrm{m} / \mathrm{ps})$, and the relative depth $\frac{n_{s}}{n_{0}}$ is found to be larger (see Fig. 3). This is consistent with our general observation that larger velocities are found to be related to smaller values of $\frac{n_{s}}{n_{0}}$. All these observations are supported by numerical simulations performed by solving iteratively the generalized dissipative Gross-Pitaevskii equation for the lower polariton branch. ${ }^{21}$ Simulations allow to reproduce the polariton fluid dynamics and DS nucleation for different positions of the polariton injection. ${ }^{13,15}$ The extracted relation between the formation velocities and the density ratio is consistent with the one observed in the experiments as well as the dependency of $v_{\text {form }}$ on the distance. This is shown with red dots in the inset of Fig. 3. Another approach for understanding the dynamics of DS formation may be provided by considering the convective instability of $\mathrm{DS}^{22}$ For values of $M$ larger than a critical one, DS instability passes from absolute to convective. After a perturbation is applied to the DS, it propagates along the

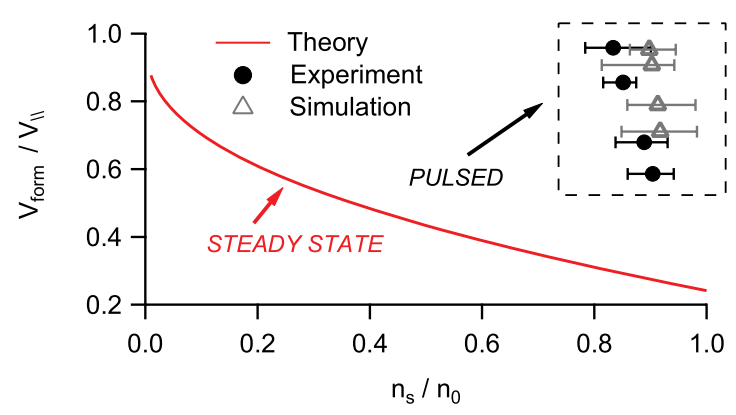

FIG. 4. (Color online) Dependence of the formation velocity on the soliton relative amplitude $\frac{n_{s}}{n_{0}}$ calculated according to the theory of Ref. 23 (red line), measured experimentally (black dots) and numerically simulated (triangles). 
soliton and can break it with the formation of vortices. ${ }^{15}$ In the case of stationary and nondissipative flow past an obstacle, it has been proven that this disturbance is convected away when the projection of the flow velocity on the soliton $v_{\|}$is larger than the propagation speed of the disturbance $v_{\mathrm{cr}}$. ${ }^{22}$ This is the case of convective instability for DSs, and the length of an oblique dark soliton is found to grow in the reference frame of the obstacle with $v_{\text {form }}=v_{\|}-v_{\text {cr }}$. In polariton systems, which are strongly dissipative, when a continuous flow is injected in the vicinity of an obstacle, its density outside the pumping region shows a gradient due to the finite lifetime of polaritons. This property changes the conditions for $v_{\mathrm{cr}}$. In Ref. 23 a formula for $v_{\text {cr }}$ has been extracted under the assumption that the density distribution does not change too quickly as it goes further from the pumping area, and it is found to be proportional to the total fluid density $n_{0}$ and the ratio $\frac{v_{s}}{c_{s}}$. Figure 4 shows the theoretical tendency of the formation velocity on the ratio $\frac{n_{0}}{n_{s}}$ for the case of a continuous injection of polaritons. This allows for a comparison with our experimental results (black dots) and the numerical simulations (triangles), which are obtained from the inset of Fig. 3. The horizontal bars indicate the range of values of $\frac{n_{s}}{n_{0}}$ covered during the soliton time window. The same trend can be observed in the three cases: a decreasing formation velocity for an increasing ration $\frac{n_{s}}{n_{0}}$. Quantitatively, our experimental and simulated values are larger than the ones predicted by the theory of Ref. 23. This difference is most likely due to the fact that the theory of Ref. 23 considers continuous wave (cw) pumping, leading to a steady state, while our experiments and simulations are carried with a pulsed excitation. Intuitively, further losses in the polariton density make $v_{\text {cr }}$ smaller, resulting in a higher formation velocity compared to the one predicted in the continuous pumping scenario.

In conclusion, we have reported on the formation dynamics of oblique dark solitons nucleated in the wake of an engineered potential in a polariton quantum fluid. The dynamics of DS formation can be controlled by tuning the fluid density distribution. In our case this has been achieved by positioning the laser spot at different distances from the obstacle. We have measured the velocity of formation of DS and have observed that the closer the polaritons are injected to the obstacle, the slower the DSs are formed, in agreement with numerical simulations based on the Gross-Pitaevskii equation. We have related the formation velocities to local quantitative measurements of the ratio $n_{s} / n_{0}$, which allow to interpret the formation velocity in the framework of convective instabilities. ${ }^{22,23}$

We acknowledge continuous support from the Swiss National Science Foundation through the NCCR Quantum Photonics Center and Project No. 135003. Complementary funding for conferences and meetings has been obtained through the Latsis Foundation, the Polatom network of the European Science Foundation, and King Saud University.
*Present address: JILA, University of Colorado, and National Institute of Standards and Technology, Boulder, CO 80309-0440.

${ }^{\dagger}$ Present address: Foton Laboratory, CNRS UMR6082, INSA Rennes, 20 Ave des buttes de Coësmes, CS14315, 35043 Rennes Cedex, France.

${ }^{1}$ T. Frisch, Y. Pomeau, and S. Rica, Phys. Rev. Lett. 69, 1644 (1992). ${ }^{2}$ G. A. El, A. Gammal, and A. M. Kamchatnov, Phys. Rev. Lett. 97, 180405 (2006).

${ }^{3}$ L. E. Sadler, J. M. Higbie, S. R. Leslie, M. Vengalattore, and D. M. Stamper-Kurn, Nature (London) 443, 312 (2006).

${ }^{4}$ A. Amo, S. Pigeon, D. Sanvitto, V. G. Sala, R. Hivet, I. Carusotto, F. Pisanello, G. Lemenager, R. Houdre, E. Giacobino et al., Science 332, 1167 (2011).

${ }^{5}$ M. Sich, D. Krizhanovskii, M. Skolnick, A. Gorbach, R. Hartley, D. Skryabin, E. Cerda-Mendez, K. Biermann, R. Hey, and P. V. Santos, Nat. Photonics 6, 50 (2011).

${ }^{6}$ T. Tsuzuki, J. Low Temp. Phys. 4, 441 (1971).

${ }^{7}$ Y. S. Kivshar, IEEE J. Quantum Electron. 29, 250 (1993).

${ }^{8}$ J. Denschlag, J. E. Simsarian, D. L. Feder, C. W. Clark, L. A. Collins, J. Cubizolles, L. Deng, E. W. Hagley, K. Helmerson, W. P. Reinhardt et al., Science 287, 97 (2000).

${ }^{9}$ B. P. Anderson, P. C. Haljan, C. A. Regal, D. L. Feder, L. A. Collins, C. W. Clark, and E. A. Cornell, Phys. Rev. Lett. 86, 2926 (2001).

${ }^{10}$ L. Pitaeskii and S. Stringari, Bose-Einstein Condensation (Oxford University Press, New York, 2003).

${ }^{11}$ J. Kasprzak, M. Richard, S. Kundermann, A. Baas, P. Jeambrun, J. M. J. Keeling, F. M. Marchetti, M. H. Szymańska, R. Andre, J. L. Staehli et al., Nature (London) 443, 409 (2006).
${ }^{12}$ A. Amo, J. Lefrère, S. Pigeon, C. Adrados, C. Ciuti, I. Carusotto, R. Houdré, E. Giacobino, and A. Bramati, Nat. Phys. 5, 805 (2009).

${ }^{13}$ G. Nardin, G. Grosso, Y. Léger, B. Piętka, F. Morier-Genoud, and B. Deveaud-Plédran, Nat. Phys. 7, 635 (2011).

${ }^{14}$ D. Sanvitto, S. Pigeon, A. Amo, D. Ballarini, M. D. Giorgi, I. Carusotto, R. Hivet, F. Pisanello, V. Sala, P. Guimaraes et al., Nat. Photonics 5, 610 (2011).

${ }^{15}$ G. Grosso, G. Nardin, F. Morier-Genoud, Y. Léger, and B. DeveaudPlédran, Phys. Rev. Lett. 107, 245301 (2011).

${ }^{16}$ G. Nardin, Y. Léger, B. Piętka, F. Morier-Genoud, and B. DeveaudPlédran, J. Nanophotonics 5, 053517 (2011).

${ }^{17}$ S. Pigeon, I. Carusotto, and C. Ciuti, Phys. Rev. B 83, 144513 (2011).

${ }^{18}$ R. I. Kaitouni, O. E. Daï, A. Baas, M. Richard, T. Paraiso, P. Lugan, T. Guillet, F. Morier-Genoud, J. D. Ganière, J. L. Staehli et al., Phys. Rev. B 74, 155311 (2006).

${ }^{19}$ Y. G. Gladush, A. M. Kamchatnov, Z. Shi, P. G. Kevrekidis, D. J. Frantzeskakis, and B. A. Malomed, Phys. Rev. A 79, 033623 (2009).

${ }^{20}$ R. Cerna, D. Sarchi, T. K. Paraïso, G. Nardin, Y. Léger, M. Richard, B. Pietka, O. E. Daïf, F. Morier-Genoud, V. Savona et al., Phys. Rev. B 80, 121309 (2009).

${ }^{21}$ I. Carusotto and C. Ciuti, Phys. Rev. Lett. 93, 166401 (2004).

${ }^{22}$ A. Kamchatnov and S. Korneev, Phys. Lett. A 375, 2577 (2011).

${ }^{23}$ A. M. Kamchatnov and S. V. Korneev, arXiv:1111.4170. 